\title{
Deformations of the Bifurcation Diagram Due to Discretization
}

\author{
By J. Bigge and E. Bohl
}

\begin{abstract}
With a singular perturbation problem occurring in chemical reaction processes, substantial changes of the bifurcation diagram due to discretization are demonstrated. It is shown that a discrete system can possess any number of solutions, whereas the underlying continuous problem has exactly one solution. In addition to that, there is no way to favor one of the various discrete solutions as the one approximating the continuous solution.
\end{abstract}

1. Introduction. It is well-known that the steady state of evolution processes in one space dimension is described by an ordinary boundary value problem of the type

$$
\begin{aligned}
-x^{\prime \prime}+\nu x^{\prime}+\mu f(\lambda, x) & =0 \text { on }[0,1], \\
x(0)=x(1) & =w .
\end{aligned}
$$

For transport phenomena arising in chemistry or biology $x^{\prime \prime}, \nu x^{\prime}$ and $\mu f(\lambda, x)$ represent diffusion, convection, and generation, respectively. In these applications the real parameters $\nu, \mu$ and $w$ naturally satisfy

$$
\nu \geqslant 0, \quad \mu \geqslant 0, \quad w \geqslant 0 .
$$

Furthermore, $\lambda$ stands for a parameter vector in $\mathbf{R}^{m}$ with nonnegative components

$$
\lambda_{i} \geqslant 0, \quad i=1, \ldots, m \text {. }
$$

The various constants may be viewed as control parameters. A typical problem is to describe the solution set of (1) with respect to some of these control parameters keeping all the others fixed. We call bifurcation diagram any two-dimensional representation of the solution set where the actual control parameter serves as abscissa and a functional of the corresponding solution as the ordinate. Often, details of the global picture of the resulting bifurcation diagram are wanted [7], [8], [11]. It often happens that general bifurcation theory cannot provide the information and numerical techniques are used. Standard discretizations are being applied on a grid consisting of a moderate number of grid points, say 10 to 20 . Retreating to numerical analysis techniques, one tacitly hopes that the continuous situation is represented in a reasonable way. However, a number of recent papers, e.g., [1], [2], [3], [5], [13], show that the bifurcation diagram of a problem of the form (1) may undergo deformations if one passes over to discrete analogues of (1). As a result, the

Received March 15, 1982; revised June 13, 1983 and February 18, 1985.

1980 Mathematics Subject Classification. Primary 65H10, 65L10, 92A05, 92A40.

Key words and phrases. Discrete deformations of bifurcation diagrams. 
diagram of the discrete problem may even fail to show qualitatively the correct response of the equation (1) to alterations in the control parameters $\nu, \mu$ or $\lambda$.

In Section 3 of this paper we describe conditions on $f(\lambda, x)$ such that standard discrete versions of (1) admit a number of solutions which depends on the qualitative behavior of $f$ and upon the ratio of the control parameter and the step width of the underlying grid. The idea is very elementary and suggests that the phenomenon of multiple solutions is not a matter of a particular finite-difference approximation for fairly general nonlinearities $f$. It rather is a natural phenomenon due to the process of substituting (1) by a finite-dimensional system. This process introduces a new parameter $h$ ( = the step width) which has the effect of a sort of "unfolding", giving rise to dramatic changes of the bifurcation diagram of (1). We begin in Section 2 with numerical examples using functions $f(\lambda, x)$ which occur in biological or chemical reaction processes.

2. Numerical Examples. Consider the problem

$$
\begin{gathered}
-x^{\prime \prime}+\nu x^{\prime}+\mu \frac{x+\lambda_{2} x^{2}}{1+x+\lambda_{1} x^{2}+\lambda_{2} x^{3}}=0 \text { on }[0,1], \\
x(0)=x(1)=w .
\end{gathered}
$$

The generation term appearing in (2a) is quite common in enzyme kinetics: for $\lambda_{1}=\lambda_{2}=0$ it describes the usual Michaelis-Menten kinetics, for $\lambda_{2}=0$ we have a substrate inhibited process, and the general expression arises under even more complicated conditions of three intermediate enzyme-substrate complexes [5b], [7], [8].

To any set of parameters $\nu, \mu, w, \lambda_{i} \geqslant 0(i=1,2)$ there exists a solution $\bar{x}$ of (2) satisfying

$$
0<\bar{x}(s)<w \quad \text { on }(0,1) \text { or } \bar{x} \equiv w .
$$

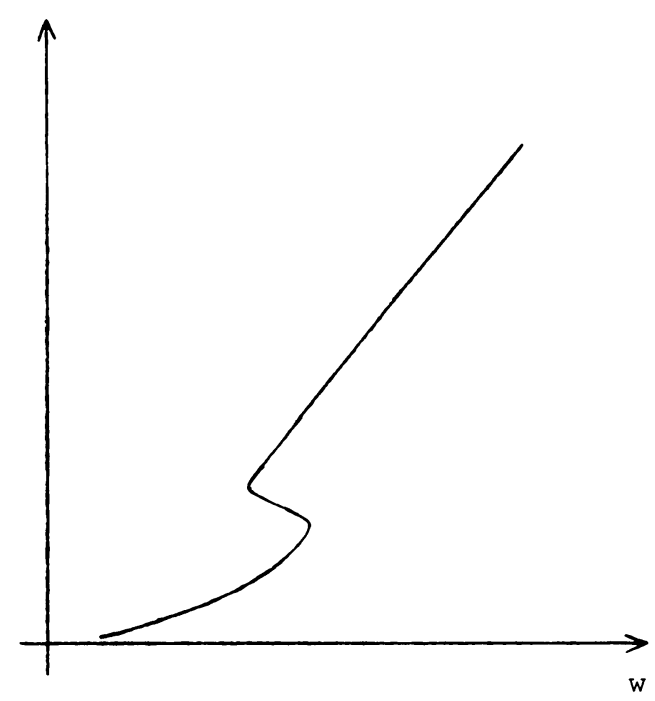

FIGURE 1

Qualitative bifurcation diagram for problem $(2)\left(\lambda_{1} \neq 0\right.$ or $\left.\lambda_{2} \neq 0\right)$ 
This is a consequence of maximum principle techniques as outlined in [9], [14]. Only solutions satisfying the a priori bounds (3) are of interest in the applications. If $\lambda_{1}=\lambda_{2}=0$, there is exactly one solution with (3) and this is also true for the standard second-order finite-difference approximation of (2). Here, again, the maximum principle may be applied (see [5b]). There are further finite-difference approximations of higher order amenable to the application of the maximum principle and thus yielding the existence and uniqueness result stated (see [5b]). As a consequence, in these cases there is no qualitative difference of the bifurcation diagram between the continuous and the discrete case with respect to any of the parameters $w, \nu$ or $\mu$ left in the equation. If, however, $\lambda_{1} \neq 0$ or $\lambda_{2} \neq 0$, there are, in general, more than one solution of (2). A qualitative picture of the bifurcation diagram is given in Figure $1(w=$ control parameter, the ordinate in Figure 1 represents the value of a norm of the solutions on the branch).

What has been outlined in the previous paragraph holds also for our second example

$$
\begin{gathered}
-x^{\prime \prime}+\nu x^{\prime}+\mu(x-1) \exp \left(-\frac{\lambda_{1}}{1+x}\right)=0, \\
x(0)=x(1)=0 .
\end{gathered}
$$

The a priori bound (3), however, must be replaced by

$$
0<\bar{x}(s)<1 \text { on }(0,1) \text {. }
$$

The generation term of (4) is known from a first-order exothermic chemical reaction [5b].

Our numerical calculations are based on the finite-difference substitutions

$$
\begin{gathered}
x^{\prime}(s) \sim h^{-1}(-x(s-h)+x(s)), \\
x^{\prime \prime}(s) \sim h^{-2}(x(s-h)-2 x(s)+x(s+h)) .
\end{gathered}
$$

The following results are obtained on an equidistant grid of 9 grid points in $(0,1)$ (i.e., $h=0.1$ ).

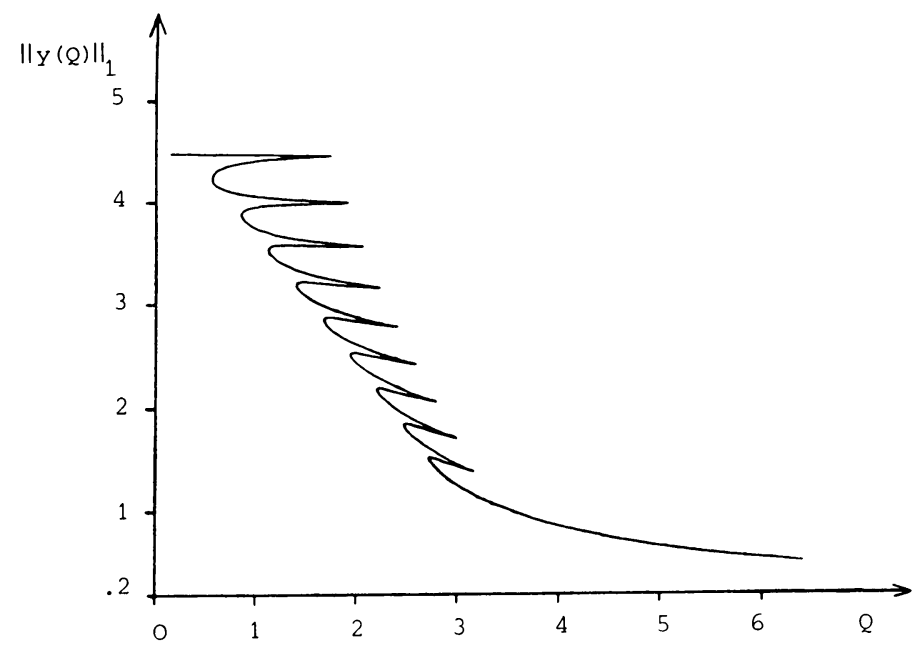

Figure 2

Solution branch of Example (2) 
TABLE 1

Turning points of the branch of Example (2)

\begin{tabular}{l|lllllll}
$Q$ & 0.1 & 1.717 & 0.523 & 1.886 & 0.820 & 2.046 & 1.103 \\
\hline$\|y(Q)\|_{1}$ & 4.499 & 4.481 & 4.247 & 4.022 & 3.907 & 3.591 & 3.561 \\
$Q$ & 2.217 & 1.381 & 2.396 & 1.655 & 2.585 & 1.927 & 2.782 \\
\hline$\|y(Q)\|_{1}$ & 3.185 & 3.207 & 2.799 & 2.880 & 2.431 & 2.521 & 2.073 \\
$Q$ & 2.195 & 2.987 & 2.464 & 3.170 & 2.712 & 6.180 & \\
\hline$\|y(Q)\|_{1}$ & 2.190 & 1.724 & 1.855 & 1.393 & 1.528 & 0.526 &
\end{tabular}

TABLE 2

\begin{tabular}{c|ccccccccc}
\multicolumn{10}{c}{9 solutions of Example (2) for $Q=2$} \\
$t$ & 0.1 & 0.2 & 0.3 & 0.4 & 0.5 & 0.6 & 0.7 & 0.8 & 0.9 \\
\hline$y(Q, t)$ & 0.3565 & 0.7844 & 4.850 & 4.997 & & & & & \\
& 0.3565 & 0.7833 & 4.751 & 4.995 & & & & & \\
& 0.3561 & 0.7448 & 1.212 & 4.891 & 4.997 & & & & \\
& 0.3561 & 0.7448 & 1.209 & 4.647 & 4.992 & & & & \\
& 0.3561 & 0.7444 & 1.176 & 1.702 & 4.915 & 4.998 & & & \\
& 0.3561 & 0.7444 & 1.176 & 1.697 & 4.515 & 4.990 & & & \\
& 0.3561 & 0.7444 & 1.176 & 1.671 & 2.298 & 4.937 & 4.998 & & \\
& 0.3561 & 0.7444 & 1.176 & 1.671 & 2.289 & 4.215 & 4.984 & & \\
& 0.3561 & 0.7444 & 1.176 & 1.671 & 2.276 & 3.172 & 4.960 & 4.999 &
\end{tabular}

Example (2): $\lambda_{1}=30, \lambda_{2}=0, \nu=10^{3}, w=5$ and $\mu=10^{6} Q^{-1}$ with $Q=$ control parameter. The results of the numerical experiment are given in Figure 2. Table 1 contains all turning points of the branch and Table 2 shows all solutions for $Q=2$ (i.e., $2 \mu=10^{6}$ ). Note that all results correspond to $y=w-x=5-x$ rather than to $x$. The blanks in Table 2 stand for function values $\geqslant 4.999$ and $<5.0$. Here and in the following we use the norm

$$
\|y(Q)\|_{1}=h \sum_{s}|y(Q, s)|
$$

where $s$ runs over the grid points. $y(Q, s)$ stands for the $s$ th component of the vector $y(Q)$.

Example (4): $\mu=10^{12}, \nu=10^{3}$ and $\lambda_{1}=$ control parameter. The results of this experiment are summarized in Figure 3. Again, Table 3 shows all turning points of the branch and Table 4 gives all solutions for $\lambda_{1}=24$. The blanks in Table 4 stand for function values $\geqslant 0.999$ and $<1$.

Both examples show more than just one hysteresis loop and consequently more than two singular points on the branch. The theory outlined in the next section will tell us that, depending upon the ratio of the control parameter and the step width, any number of solutions (of hysteresis loops) may be constructed. All of them satisfy the qualitative characteristics (one maximum point, a priori bounds etc.) suggested by the applications (biology or chemistry) so that, from merely glancing at the figures, there is no way of ruling out one of these solutions against others. Also, stability would not favor just two steady states: stable and unstable parts of the branches take turns at any turning point. 
TABLE 3

Turning points of the branch of Example (4)

\begin{tabular}{c|cccccccc}
$\lambda_{1}$ & 18.00 & 30.87 & 22.60 & 30.89 & 22.92 & 30.89 & 23.15 & 30.89 \\
\hline$\left\|x\left(\lambda_{1}\right)\right\|_{1}$ & 0.899 & 0.886 & 0.806 & 0.787 & 0.709 & 0.687 & 0.612 & 0.587 \\
$\lambda_{1}$ & 23.34 & 30.89 & 23.49 & 30.89 & 23.63 & 30.89 & 23.74 & 30.89 \\
\hline$\left\|x\left(\lambda_{1}\right)\right\|_{1}$ & 0.516 & 0.487 & 0.420 & 0.386 & 0.325 & 0.287 & 0.227 & 0.187 \\
$\lambda_{1}$ & 23.85 & 30.87 & 23.91 & 34.53 & & & & \\
\cline { 1 - 3 }$\left(\lambda_{1}\right) \|_{1}$ & 0.132 & 0.087 & 0.039 & $0.4 \cdot 10^{-6}$ & & & &
\end{tabular}

TABLE 4

19 solutions of Example (4) for $\lambda_{1}=24$

\begin{tabular}{c|ccccccccc}
$t$ & 0.1 & 0.2 & 0.3 & 0.4 & 0.5 & 0.6 & 0.7 & 0.8 & 0.9 \\
\hline$x\left(\lambda_{1}, t\right)$ & 0.9983 & & & & & & & & \\
& 0.2160 & 0.9987 & & & & & & & \\
& 0.0149 & 0.9983 & & & & & & & \\
& 0.0063 & 0.2129 & 0.9986 & & & & & & \\
& 0.0042 & 0.0198 & 0.9983 & & & & & & \\
& 0.0041 & 0.0109 & 0.2105 & 0.9986 & & & & & \\
& 0.0041 & 0.0088 & 0.0250 & 0.9983 & & & & & \\
& 0.0041 & 0.0087 & 0.0160 & 0.2077 & 0.9986 & & & & \\
& 0.0041 & 0.0087 & 0.0140 & 0.0311 & 0.9983 & & & & \\
& 0.0041 & 0.0087 & 0.0139 & 0.0219 & 0.2043 & 0.9986 & & & \\
& 0.0041 & 0.0087 & 0.0139 & 0.0200 & 0.0382 & 0.9984 & & & \\
& 0.0041 & 0.0087 & 0.0139 & 0.0198 & 0.0286 & 0.2000 & 0.9986 & & \\
& 0.0041 & 0.0087 & 0.0139 & 0.0198 & 0.0268 & 0.0467 & 0.9984 & & \\
& 0.0041 & 0.0087 & 0.0139 & 0.0198 & 0.0267 & 0.0367 & 0.1943 & 0.9986 & \\
& 0.0041 & 0.0087 & 0.0139 & 0.0198 & 0.0267 & 0.0351 & 0.0574 & 0.9984 & \\
& 0.0041 & 0.0087 & 0.0139 & 0.0198 & 0.0267 & 0.0349 & 0.0467 & 0.1859 & 0.9986 \\
& 0.0041 & 0.0087 & 0.0139 & 0.0198 & 0.0267 & 0.0349 & 0.0453 & 0.0718 & 0.9984 \\
& 0.0041 & 0.0087 & 0.0139 & 0.0198 & 0.0267 & 0.0349 & 0.0451 & 0.0601 & 0.1826 \\
& 0.0041 & 0.0087 & 0.0139 & 0.0198 & 0.0267 & 0.0349 & 0.0451 & 0.0586 & 0.0771
\end{tabular}

One might conjecture that the described effect of discretization on the bifurcation diagram is due to the large convection term $10^{3} x^{\prime}$ present in both examples. Indeed, the analysis of the next section depends heavily on the presence of this term. However, we mention in passing that the discrete branch $(h=0.1)$ of Example 4 with $\mu=10^{12}, \lambda_{1}=$ control parameter and $\nu=0$ (i.e., convection does not take place) has more than two singular points, among them at least four bifurcation points. From these bifurcation points further branches of asymmetric solutions emanate. In contrast to these phenomena occurring in the discrete problem, the existence of bifurcation points on the symmetric branch belonging to the continuous problem (4) are excluded by the theory in [2].

3. Two Theorems. Consider the singular perturbation problem

$$
\begin{gathered}
-\varepsilon x^{\prime \prime}+x^{\prime}=f(\lambda, x) \text { on }[0,1], \\
x(0)=x(1)=0,
\end{gathered}
$$




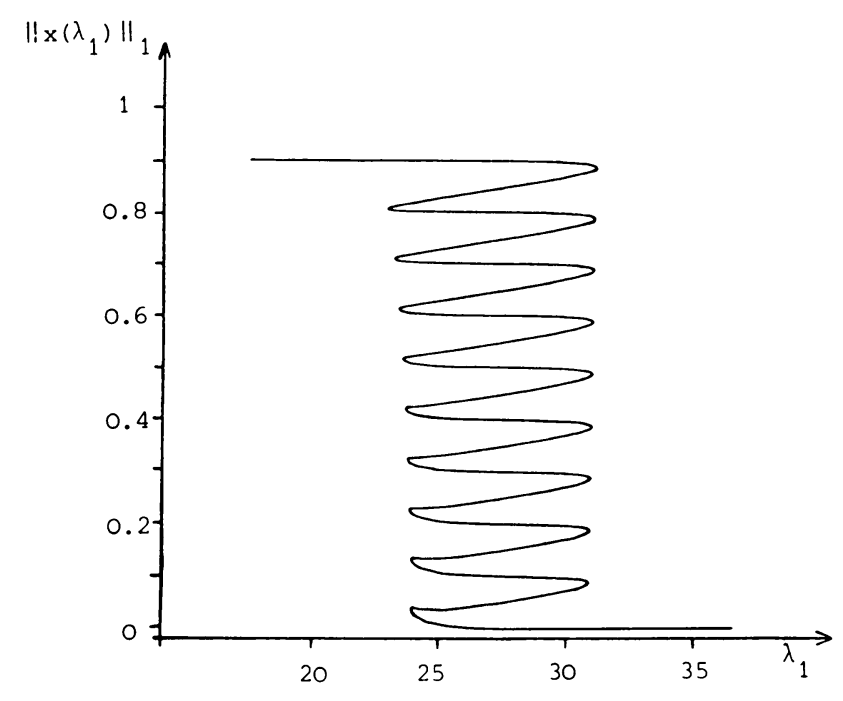

FIGURE 3

Solution branch of Example (4)

with a real control parameter $\lambda \geqslant 0$ and a small parameter $0<\varepsilon \ll 1$. Using the usual difference substitutions (6) we obtain the discrete analogue for (7)

$$
\begin{gathered}
x(0)=0, \\
h^{-2}\left(-(\varepsilon+h) x\left(s_{j-1}\right)+(2 \varepsilon+h) x\left(s_{j}\right)-\varepsilon x\left(s_{j+1}\right)\right) \\
=f\left(\lambda, x\left(s_{j}\right)\right), \quad j=1, \ldots, M, \\
x(1)=0,
\end{gathered}
$$

on the grid $\Omega_{h}=\left\{s_{j}=j h: h=(M+1)^{-1}, j=0, \ldots, M+1\right\}$. If $\varepsilon=0$, the system reduces to

$$
\begin{gathered}
x(0)=0, \\
x\left(s_{j}\right)=x\left(s_{j-1}\right)+h f\left(\lambda, x\left(s_{j}\right)\right), \quad j=1, \ldots, M,
\end{gathered}
$$

A solution of (9) is a grid function $x=\left(x(0), x\left(s_{1}\right), \ldots, x\left(s_{M}\right), x(1)\right) \in \mathbf{R}^{\Omega_{h}}$. By (9b) the value $x\left(s_{j}\right)$ marks the intersection of the graph of

$$
y \rightarrow f(\lambda, y)
$$

with the straight line

$$
y \rightarrow h^{-1}\left(y-x\left(s_{j-1}\right)\right)
$$

This process defines one component of $x$ at a time as shown in Figure 4. If the graph of $f$ and the slope $h^{-1}$ of the straight lines (11) allow for more than one intersection point, the possibility of more than just one solution to the system (9) 


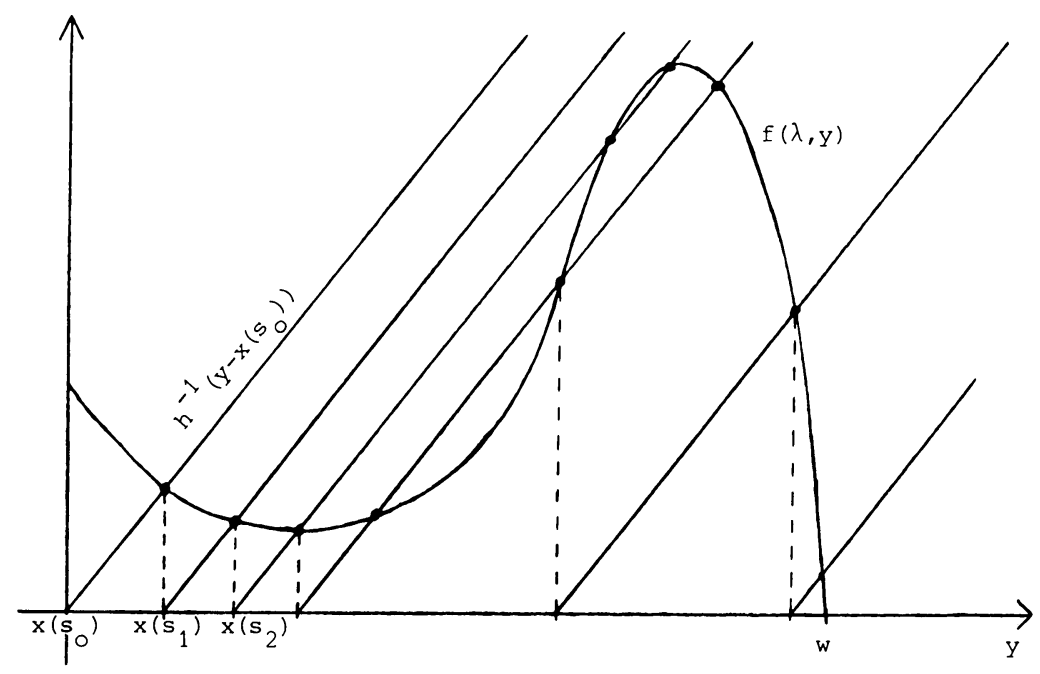

FigURE 4

Intersections of the graph of $f$ with the straight lines (11)

arises. Clearly, the number of solutions then depends upon how many of the straight lines (11) intersect the graph of (10) how many times. A graph of $f$ qualitatively given in Figure 4 allows straight lines of the form (11) to intersect in one, two or three points. Such a graph is ensured by the following assumptions f1 and $\mathrm{f} 2$. Let $0 \leqslant \beta_{1}<\beta_{2}, 0<w$ and let $U \subset \mathbf{R}^{2}$ be an open set containing $\left[\beta_{1}, \beta_{2}\right] \times[0, w]$ :

f1: $f \in C^{\infty}(U), f(\lambda, w)=0$ for $\lambda \in\left[\beta_{1}, \beta_{2}\right]$, $f(\lambda, x)>0$ for $\beta_{1} \leqslant \lambda \leqslant \beta_{2}, 0 \leqslant x<w, f_{\lambda}(\lambda, x)<0$ for $\beta_{1} \leqslant \lambda \leqslant \beta_{2}, 0<$ $x<w$.

f2: There exists a real nondecreasing function $u(\lambda)$ on $\left[\beta_{1}, \beta_{2}\right]$ such that $0 \leqslant u(\lambda)<w, f_{x x x}(\lambda, u(\lambda))<0$ for $\beta_{1} \leqslant \lambda \leqslant \beta_{2}$, and

$$
f_{x x}(\lambda, x) \begin{cases}>0 & \text { for } 0<x<u(\lambda), \\ <0 & \text { for } u(\lambda)<x<w .\end{cases}
$$

f3: There exist real numbers $\gamma_{1}, \gamma_{2}, \beta_{1} \leqslant \gamma_{1}<\gamma_{2}<\beta_{2}$ such that the following is true:

(a) for $\beta_{1} \leqslant \lambda \leqslant \gamma_{1}$ all solutions $\bar{y}$ of $y=h f(\lambda, y)$ satisfy $\bar{y} \in(u(\lambda), w]$.

(b) $\quad h f_{x}(\lambda, u(\lambda)) \begin{cases}>1 & \text { for } \gamma_{1} \leqslant \lambda<\gamma_{2} \\ <1 & \text { for } \gamma_{2}<\lambda \leqslant \beta_{2}\end{cases}$

The assumption f3 serves two purposes: Part (a) ensures that all solutions of (9) for $\lambda$ near $\beta_{1}$ have components close to $w$. On the other hand, (b) implies

$$
h f_{x}\left(\gamma_{2}, u\left(\gamma_{2}\right)\right)=1
$$

so that any straight line (11) intersects the graph (10) for $\lambda=\gamma_{2}$ just once. The process of solving our system (9) in this particular situation is indicated in Figure 5. 


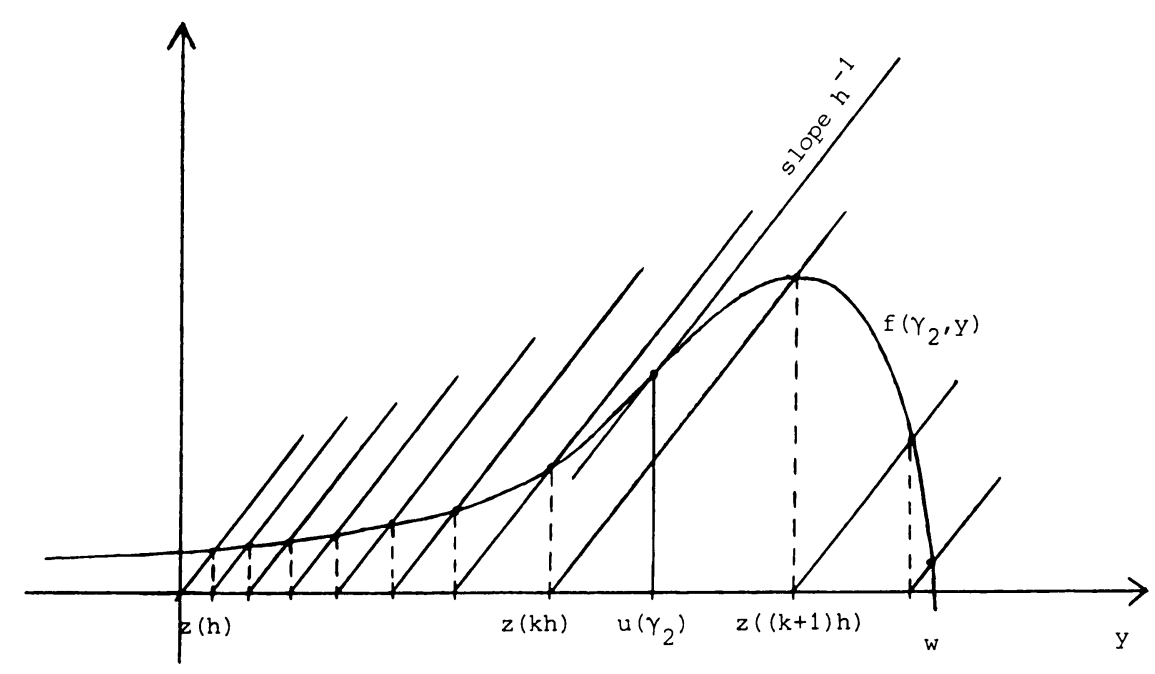

Figure 5

Determination of the number $k$ due to (12), (13). Here: $k=7$

Let this process yield the solution $\left(\gamma_{2}, z\right)$, where $z$ has the components $z(j h)$ $(j=0, \ldots, M+1)$. Then a natural number $k$ is uniquely determined by

$$
z(j h)<u\left(\gamma_{2}\right) \quad(j=1, \ldots, k), \quad u\left(\gamma_{2}\right) \leqslant z((k+1) h) .
$$

This number $k$ will define the number of hysteresis loops exhibited by a solution branch of (9), as stated in the following theorem.

THEOREM 1. Let $h>0$ be fixed. Under the assumptions $\mathrm{f} 1-\mathrm{f} 3$ there exists a smooth $\left(=C^{\infty}\right)$ branch $(\lambda(\sigma), x(\sigma))(\sigma \in[0,1])$ of solutions of $(9)$ such that

(a) $0<x(\sigma, s)<w$ for $\sigma \in[0,1], s \in \Omega_{h} \backslash\{0,1\}$.

(b) $\lambda(\sigma) \neq \lambda(\tau)$ or $x(\sigma) \neq x(\tau)(\sigma \neq \tau, \sigma, \tau \in[0,1]), \lambda(0)=\beta_{1}, \lambda(1)=\beta_{2}$.

(c) For $\lambda \in\left[\beta_{1}, \beta_{2}\right]$ all solutions of (9) subject to the a priori bound given in (a) are on the branch $(\lambda(\sigma), x(\sigma))$.

(d) The function $\lambda(\sigma)$ has exactly $2 k$ relative extreme points in $(0,1)$ where $k$ is defined by (13) above.

Remark. By the assertion (d), the branch $(\lambda(\sigma), x(\sigma))$ shows $k$ hysteresis loops in the $(\lambda, x)$-diagram. Hence, the system (9) may have up to $2 k+1$ different solutions for some parameter $\lambda=\lambda(\sigma)$.

As an illustration, take the system (2) with $\lambda_{2}=0$. In the new variable $y=w-x$ the nonlinearity has the form

$$
f(\lambda, y)=10^{6} \lambda^{-1} \frac{w-y}{1+(w-y)+30(w-y)^{2}} .
$$

The graph of this function is qualitatively given in Figure 5. The function $u(\lambda, w)$ mentioned in assumption $\mathrm{f} 2$ depends on $w$. It turns out that for any fixed $\lambda$, this function monotonically approaches infinity for increasing $w$. We can choose the interval $[0, u(\lambda, w)]$ as long as we want if we increase the parameter $w$. Proceed now as follows: Fix $h>0$ and find $\gamma_{2}$ such that (12) is satisfied. By an elementary 
discussion of the graph of $f$ in (14) the construction of $\gamma_{2}$ is possible, independently of $w$. Now, the process illustrated in Figure 5 may be applied $k$ times for any given $k \in \mathbf{N}$. This defines $w$. The resulting function $f$ yields a corresponding function $\lambda(\sigma)$ of Theorem 1 which has exactly $2 k$ relative extreme points in $(0,1)$.

Sketch of the Proof of Theorem 1 (for details see [4]): Let $I=\left[\beta_{1}, \beta_{2}\right], D=\{(\lambda, x)$ $\in I \times \mathbf{R}^{\Omega_{h}}: 0 \leqslant x\left(s_{j}\right) \leqslant w$ for $\left.\left.j=1, \ldots, M\right)\right\}$ and let the matrix $A \in L\left[\mathbf{R}^{\Omega_{h}}\right]$ be defined by the left-hand side of (8) for $\varepsilon=0$. The right-hand side of (8) defines the function $F(\lambda, x)$ which maps $D$ into $\mathbf{R}^{\Omega_{h}}$. The step width $h$ is fixed. Finally, the operator $T(\lambda, x)=A x-F(\lambda, x)$ is defined on $D$ and has values in $\mathbf{R}^{\Omega_{h}}$. The objective is to describe the set $T^{-1}(\theta)=\{(\lambda, x) \in D: T(\lambda, x)=\theta\}$. It is a technical matter to see that under our assumptions $T^{-1}(\theta)$ is a one-dimensional differentiable manifold with boundary (see Section 2, Lemma 4 of [12]). Since the equations

$$
T\left(\beta_{i}, x\right)=0 \quad(i=1,2)
$$

have a unique solution $x^{i}(i=1,2)$, the boundary $\partial T^{-1}(\theta)$ of $T^{-1}(\theta)$ is given by

$$
\partial T^{-1}(\theta)=\left\{\left(\beta_{1}, x^{1}\right),\left(\beta_{2}, x^{2}\right)\right\} .
$$

Furthermore, we learn from the Theorem in the appendix of [12], that the compact set $T^{-1}(\theta)$ is a disjoint union of finitely many connected components, and any of these components is either diffeomorphic to $[0,1]$ or to the unit circle $S^{1}$ in the plane. Since there is a unique solution of the equations (15), there is exactly one component of $T^{-1}(\theta)$ which is diffeomorphic to [0,1]. This component represents a $C^{\infty}$-solution branch which we write in the form

$$
(\lambda(\sigma), x(\sigma)), \quad \sigma \in[0,1] .
$$

This branch satisfies our statements (a) and (b) of Theorem 1. The corresponding function $\lambda(\sigma)$ has Property (d). Indeed, let $k$ be the number defined in (13), where the vector $z$ represents the unique solution of the system $T\left(\gamma_{2}, x\right)=\theta$. Then, one can show for any $j \in\{1, \ldots, k\}$, there are exactly two parameter values $\sigma_{1 j}, \sigma_{2 j} \in(0,1)$ such that

$$
\lambda^{\prime}\left(\sigma_{1 j}\right)=\lambda^{\prime}\left(\sigma_{2 j}\right)=0, \quad \lambda^{\prime \prime}\left(\sigma_{1 j}\right) \neq 0, \quad \lambda^{\prime \prime}\left(\sigma_{2 j}\right) \neq 0, \quad j=1, \ldots, k .
$$

Since $\sigma_{i r} \neq \sigma_{p q}(i \neq p$ or $r \neq q)$ we have constructed $2 k$ different extreme points of the function $\lambda(\sigma)$ and these are all extreme points of $\lambda(\sigma)$ in $(0,1)$.

To complete the proof of Theorem 1 we must exclude the possibility of components diffeomorphic to $S^{1}$ in the set $T^{-1}(\theta)$. This is a rather lengthy and technical argument which we omit here.

THEOREM 2. Under the assumptions $\mathrm{f} 1-\mathrm{f} 3$ there exists $\varepsilon_{0}>0$ such that for any $\varepsilon \in\left[0, \varepsilon_{0}\right]$ the full problem (8) admits a branch of solutions $(\lambda(\varepsilon, \sigma), x(\varepsilon, \sigma)), \sigma \in[0,1]$ such that

(a) $\lambda(\varepsilon, \sigma), x(\varepsilon, \sigma)$ are $C^{\infty}$-functions on $\left[0, \varepsilon_{0}\right] \times[0,1]$.

(b) $0<x(\varepsilon, \sigma, s)<w$ for $\varepsilon \in\left[0, \varepsilon_{0}\right], \quad \sigma \in[0,1], s \in \Omega_{h} \backslash\{0,1\}, \lambda(\varepsilon, 0)=$ $\beta_{1}, \lambda(\varepsilon, 1)=\beta_{2}$.

(c) For $\lambda \in\left[\beta_{1}, \beta_{2}\right]$ all solutions of (8) subject to the a priori bound given in (b) are on the branch $(\lambda(\varepsilon, \sigma), x(\varepsilon, \sigma))$. 
(d) $\lambda(\varepsilon, \sigma) \rightarrow \lambda(\sigma), x(\varepsilon, \sigma) \rightarrow x(\sigma)$ as $\varepsilon \rightarrow 0$, where $(\lambda(\sigma), x(\sigma))$ is the solution branch of (9) described in Theorem 1 .

(e) For any $\varepsilon \in\left[0, \varepsilon_{0}\right]$ the function $\lambda(\varepsilon, \sigma)$ has at least $2 k$ relative extreme points in $(0,1)$ where $k$ is the number defined by (13) and referred to in (d) of Theorem 1 . More precisely, any of the $2 k$ extreme points of the function $\lambda(\sigma)$ of Theorem 1 produces exactly one extreme point of the perturbed function $\lambda(\varepsilon, \sigma)$.

Sketch of the Proof (for the details see [4]): The statements (a)-(d) are an immediate consequence of a perturbation argument based on the results of Theorem 1. It is a straightforward application of the implicit function theorem. This way of reasoning, of course, only yields that the perturbed branch is closed to the unperturbed branch (17). However, one can also prove a perturbed version of (18). More precisely, for any unperturbed $\sigma_{1 j}, \sigma_{2 j} \in(0,1)(j=1, \ldots, k)$, as given in (18), there exist small intervals $I\left(\sigma_{1 j}\right), I\left(\sigma_{2 j}\right) \subset[0,1](j=1, \ldots, k)$ about $\sigma_{1 j}, \sigma_{2 j}$ such that for any $\varepsilon \in\left[0, \varepsilon_{0}\right]$ there exist unique $\sigma_{1 j}^{\varepsilon} \in I\left(\sigma_{1 j}\right), \sigma_{2 j}^{\varepsilon} \in I\left(\sigma_{2 j}\right)(j=1, \ldots, k)$ such that

$$
\begin{gathered}
\sigma_{i r}^{\varepsilon} \neq \sigma_{p q}^{\varepsilon} \quad(i \neq p, r \neq q), \quad \frac{\partial}{\partial \sigma} \lambda\left(\varepsilon, \sigma_{1 j}^{\varepsilon}\right)=\frac{\partial}{\partial \sigma} \lambda\left(\varepsilon, \sigma_{2 j}^{\varepsilon}\right)=0, \\
\frac{\partial^{2}}{\partial \sigma^{2}} \lambda(\varepsilon, \sigma) \neq 0 \quad \text { for } \sigma \in I\left(\sigma_{1, j}\right) \cup I\left(\sigma_{2, j}\right) .
\end{gathered}
$$

This means that any extreme point $\sigma_{1 j}, \sigma_{2 j}$ of $\lambda(\sigma)$ yields exactly one extreme point of $\lambda(\sigma, \varepsilon)$ for $\varepsilon \in\left[0, \varepsilon_{0}\right]$ in a small neighborhood $I\left(\sigma_{1 j}\right), I\left(\sigma_{2 j}\right)$. This is our statement (e), so that the proof of Theorem 2 is complete.

The Example (4) has also been considered by H. O. Kreiss. He has used his grid-finding procedure [10], which could find only one solution for some $\lambda_{1} \in(26,30)$. Grid-finding procedures are naturally only aimed at approximate solutions of the continuous problem, which has exactly one solution in Example (4). The grid-finding procedure excludes the many solutions of the discrete system on a fixed grid due to the fact that the spurious solutions are normally very sensitive to a change of the grid. Kreiss' findings suggest that some variation of the grid is important for a sensible numerical treatment of nonlinear boundary value problems. Example (2) provides an interesting situation for $\nu=0, \lambda_{1}=30, \lambda_{2}=36, \mu=10^{3}$ and $w=$ control parameter. The resulting branch shows at least 10 turning points on a grid of 9 points in $(0,1)(h=0.1)$. If we go for 39 grid points in $(0,1)$ (i.e., $h=0.025)$, then the branch is smoothed out with only 2 turning points left. We stress that, with substantial influence of a convection term (as in the examples of Section 2), one would need far more equidistant grid points to achieve a bifurcation diagram which resembles the one of the continuous problem at least qualitatively. In the case of the Example (4) we would have to allow for as many as $10^{7}$ equidistant points, assuming the discrete model (8).

Fakultät für Mathematik

Universität Konstanz

Universitätstrasse 10

D-7750 Konstanz, West Germany 
1. E. Allgower, "On a discretization of $y^{\prime \prime}+\lambda y^{h}=0$," in Proc. Conf. Roy. Irish Acad. (J. J. H. Miller, ed.), Academic Press, New York, 1975.

2. W.-J. BEYN, Lösungszweige nichtlinearer Randwertaufgaben und ihre Approximation mit dem Differenzenverfahren, Habilitationsschrift, Universität Konstanz, 1981.

3. W.-J. BEYN \& J. LORENZ, "Spurious solutions for discrete superlinear boundary value problems," Computing, v. 28, 1982, pp. 43-51.

4. J. BigGE, Lösungsweige von Diskretisierungen nichtlinearer Randwertaufgaben, $\mathrm{Ph} . \mathrm{D}$. Thesis, Universität Konstanz, 1984.

5a. E. BоHL, "On the bifurcation diagram of discrete analogues for ordinary bifurcation problems," Math. Methods Appl. Sci., v. 1, 1979, pp. 566-671. 1981.

5b. E. BоHL, Finite Modelle gewöhnlicher Randwertaufgaben, LAMM-51, Teubner-Verlag, Stuttgart,

6. H. B. Keller, "Some positone problems suggested by nonlinear heat generation," in Bifurcation Theory and Nonlinear Eigenvalue Problems (J. B. Keller and S. Antman, eds.), Benjamin, New York, 1969.

7. J.-P. KerneveZ, Enzyme Mathematics, Stud. Math. Appl., vol. 10, North-Holland, Amsterdam, 1980.

8. J.-P. Kernevez \& D. Thomas, "Numerical analysis of some biochemical systems," Appl. Math. Optim., v. 1, 1975, pp. 222-285.

9. H. W. KNOBLOCH, "Second order differential inequalities and a nonlinear boundary value problem," J. Differential Equations, v. 5, 1969, pp. 55-71.

10. B. KREISS \& H.-O. KREISS, "Numerical methods for singular perturbation problems," SIAM J. Numer. Anal., v. 18, 1981, pp. 262-276.

11. J. D. Murray, Lectures on Nonlinear-Differential-Equation Models in Biologv, Clarendon Press, Oxford, 1977.

12. J. W. Milnor, Topology From the Differentiable Viewpoint, University Press of Virginia, Charlottesville, 1965 .

13. H. O. Peitgen, D. Saupe \& K. Schmitt, "Nonlinear elliptic boundary value problems versus their finite difference approximations: Numerically irrelevant solutions," J. Reine Angew. Math., v. 322, 1981, pp. 74-117.

14. D. SAtTinger, "Monotone methods in nonlinear elliptic and parabolic boundary value problems," Indiana Univ. Math. J., v. 21, 1972, pp. 979-1000. 